\title{
Controlling Structural Transitions in AuAg Nanoparticles through Precise Compositional Design
}

\author{
Anna L. Gould, ${ }^{\dagger, \ddagger}$ Kevin Rossi, ${ }^{\S}$ C. Richard A. Catlow, ${ }^{\dagger,}, \|$ Francesca Baletto, ${ }^{*}, \S$ \\ and Andrew J. Logsdail $*,, \|$ \\ ${ }^{\dagger}$ University College London, Kathleen Lonsdale Materials Chemistry, Department of Chemistry, 20 Gordon Street, London WC1H \\ 0AJ, United Kingdom \\ ${ }^{\ddagger}$ The U.K. Catalysis Hub, Research Complex at Harwell, Rutherford Appleton Laboratory, Oxfordshire OX11 0FA, United Kingdom \\ ${ }^{\S}$ Physics Department, King's College London, London WC2R 2LS, United Kingdom \\ "Cardiff Catalysis Institute, School of Chemistry, Cardiff University, Cardiff CF10 3AT, United Kingdom
}

Supporting Information

\begin{abstract}
We present a study of the transitional pathways between highsymmetry structural motifs for AgAu nanoparticles, with a specific focus on controlling the energetic barriers through chemical design. We show that the barriers can be altered by careful control of the elemental composition and chemical arrangement, with core@shell and vertex-decorated arrangements being specifically influential on the barrier heights. We also highlight the complexity of the potential and free energy landscapes for systems where there are low-symmetry geometric motifs that are energetically competitive to the high-symmetry arrangements. In particular, we highlight that some core@shell arrangements preferentially transition through multistep restructuring of lowsymmetry truncated octahedra and rosette-icosahedra, instead of via the more straightforward square-diamond transformations, due to lower energy barriers and competitive energetic minima. Our results have promising implications for the continuing efforts in bespoke nanoparticle design for catalytic and plasmonic applications.
\end{abstract}

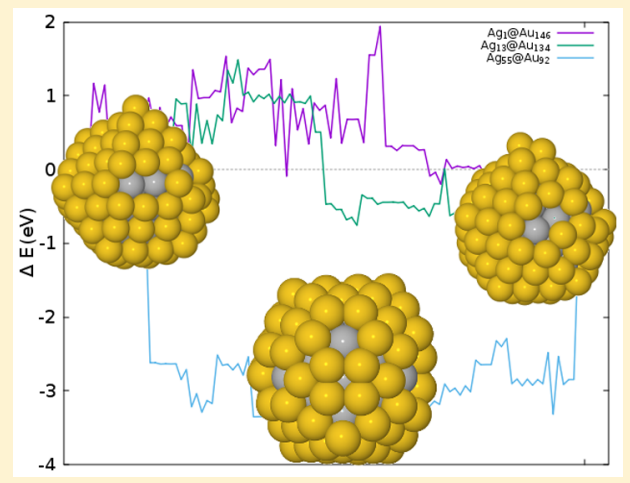

$\mathrm{N}$ anoparticles (NPs) consist of between a few and many thousands of atoms or molecules, interacting to form discrete particles. ${ }^{1}$ NPs have properties that are distinct from bulk and atomic systems, which, when considered alongside their nanoscale size, make them very suitable for applications in scientific fields such as medicine, optics, and catalysis. ${ }^{2-7}$ Recent research has shown that the nuclearity, composition and chemical ordering of bimetallic NPs can be well controlled during synthesis, ${ }^{8-14}$ but it remains an experimental challenge to control the shape of these systems. ${ }^{5,9,15}$ From an applied catalysis perspective, it would be beneficial if one could preferentially form greater quantities of reactive sites, such as vertices and edges, ${ }^{5,8,16-21}$ over unreactive sites. However, synthesis, characterization, and application of the NPs is generally performed under conditions that do not aid structure stabilization: A multitude of geometric arrangements coexist on most energy landscapes, ${ }^{8,22,23}$ possibly with similar energies and separated by transition barriers that can be overcome at room temperature. $^{15,24,25}$ The elevated operating temperatures for catalytic applications further exacerbates the problem of structural instability, and therefore understanding how to control the morphologies of NPs is of paramount importance to further their commercial applicability.
The size of NPs makes them an attractive problem to address with computational techniques, and previous work using thermodynamic sampling has identified key transition pathways and barriers between structural motifs at a range of different nuclearities, ${ }^{16,26-30}$ as well as demonstrating how the energy barriers change with chemical ordering in bimetallic NPs. ${ }^{31-34}$ One well-characterized pathway is the martensitic transformation between icosahedral (Ih) and cuboctahedral (CO) motifs via the so-called sextuple diamond-square-diamond (DSD) mechanism: ${ }^{26,35}$ Triangular facets are stretched and then rotated to form a diamond one and then transformed to a square facet ${ }^{26}$ (DS), with the reverse square-diamond process (SD) leading to reformation of the Ih from the CO. Recently, forward (SD) and backward (DS) transition pathways have been characterized at finite temperatures through molecular dynamics modeling for 147-atom $\mathrm{Ag}$ and AuAg NPs; ${ }^{32,33}$ however, no such transition occurs for Au NPs, ${ }^{32,34,36}$ showing that structural stability or transition barriers change considerably as a function of chemical composition. From a structural design perspective, controlling these transitions via chemical

Received: September 23, 2016

Accepted: October 19, 2016

Published: October 19, 2016 
methods would be ideal, but there has been limited investigation of how to use NP composition to achieve this goal. Thus we present work in this Letter that shows how the transition barriers between different geometries for bimetallic AuAg NPs can be altered by careful chemical arrangement and stoichiometric control, which could allow NP design through improved stability of certain structures.

We have focused on the transition between the Ih and $\mathrm{CO}$ motifs, which are high-symmetry NP morphologies with identical nuclearities but significant structural differences: The Ih has low internal volume and the surface is composed of 20 (111) facets, while the CO is an FCC fragment with greater volume and a mix of (100) and (111) surfaces. ${ }^{37}$ The relative stability of these motifs varies with elemental composition and also depends on the number of atoms $(N)$ in the NPs. ${ }^{8,37,38} \mathrm{We}$ have focused on the "magic" number of $N=147$, which ensures geometric closed-shells in the structures of interest. ${ }^{37}$ We have used a range of complementary modeling techniques, coupled to empirical potentials, ${ }^{32,39,40}$ to investigate transition pathways between structures, and subsequent energy barriers, in both the thermodynamic and kinetic regime. A pathway-exploring doubly nudged elastic band (DNEB) algorithm, ${ }^{41}$ as implemented in the OPTIM packages, ${ }^{42,43}$ was used for identification of transition pathways at $0 \mathrm{~K}$, while for exploration of the free-energy landscape we used the complementing discrete path-sampling (DPS) routines from OPTIM, namely, PATHSAMPLE, ${ }^{42-46}$ as well as our own metadynamics software. ${ }^{30}$ In particular, metadynamics includes anharmonic effects that are disregarded by DPS and also offers a quasi-agnostic exploration of the energy landscape due to the open-ended search of the conformational space. ${ }^{47,48}$ When not identified during the transition pathway calculations, the DS/ SD pathways between local minima were tested manually using the optimization routines in GULP. ${ }^{49}$

Initially, the stabilities of the monometallic $\mathrm{Ag}_{147}$ and $\mathrm{Au}_{147}$ NPs were calculated: DNEB pathways were identified with one barrier for the forward ( $\mathrm{CO} \rightarrow \mathrm{Ih}$ ) and backward ( $\mathrm{Ih} \rightarrow \mathrm{CO}$ ) transitions. The pathways follow the $\mathrm{SD}$ and $\mathrm{DS}$ routes, respectively, with the respective barrier energies, $\Delta E(\mathrm{CO} \rightarrow \mathrm{Ih})$ and $\Delta E(\mathrm{Ih} \rightarrow \mathrm{CO})$, being 0.50 (1.14) and 3.37 (2.33) eV for $\mathrm{Ag}_{147}\left(\mathrm{Au}_{147}\right)$.

Substitution of a single $\mathrm{Au}$ dopant in to the $\mathrm{Ag}_{147} \mathrm{NP}$ generally results in minor variation of the forward and barrier, by $\pm 0.02 \mathrm{eV}$. Exceptional changes in $\Delta E(\mathrm{Ih} \rightarrow \mathrm{CO})$ occur when the $\mathrm{Au}$ is positioned: (i) subsurface below an edge atom $(3.33 \mathrm{eV})$ and (ii) in the middle of the (111) surface facet (3.30 $\mathrm{eV}$ ). For $\mathrm{Ag}$ dopants in the $\mathrm{Au}_{147} \mathrm{NPs}$, slight variation was again observed: the barriers are generally reduced by at most $0.05 \mathrm{eV}$ from the $\mathrm{Au}_{147}$ values. An exception occurs when $\mathrm{Ag}$ is positioned at a surface vertex, with $\Delta E$ increased by $0.02 \mathrm{eV}$ in both directions; however, the transition mechanism remains identical. Ag doping in the middle of the (100) facet also reduces $\Delta E(\mathrm{CO} \rightarrow \mathrm{Ih})$ and $\Delta E(\mathrm{Ih} \rightarrow \mathrm{CO})$ by 0.06 and 0.09 $\mathrm{eV}$, respectively, to 1.08 and $2.24 \mathrm{eV}$.

A distinct variation occurs when $\mathrm{Ag}$ is positioned at the center of Au NP, akin to a core@shell arrangement. This Ag $@$ $\mathrm{Au}_{146}$ arrangement is the energetically least favorable for an $\mathrm{Ag}$ dopant, ${ }^{40}$ and we discover that the thermodynamic pathway between $\mathrm{CO}$ and Ih motifs contains 44 local minima (Figure 1, top). In this case, $\Delta E(\mathrm{CO} \rightarrow \mathrm{Ih})$ increases to $2.03 \mathrm{eV}$ and $\Delta E(\mathrm{Ih} \rightarrow \mathrm{CO})$ to $3.01 \mathrm{eV}$ compared with $\mathrm{Au}_{147}$. The transition goes through a variety of truncated octahedra (TO) and rosette-Ih (r-Ih) motifs, ${ }^{16,29}$ proceeding via sequential CO $\rightarrow$
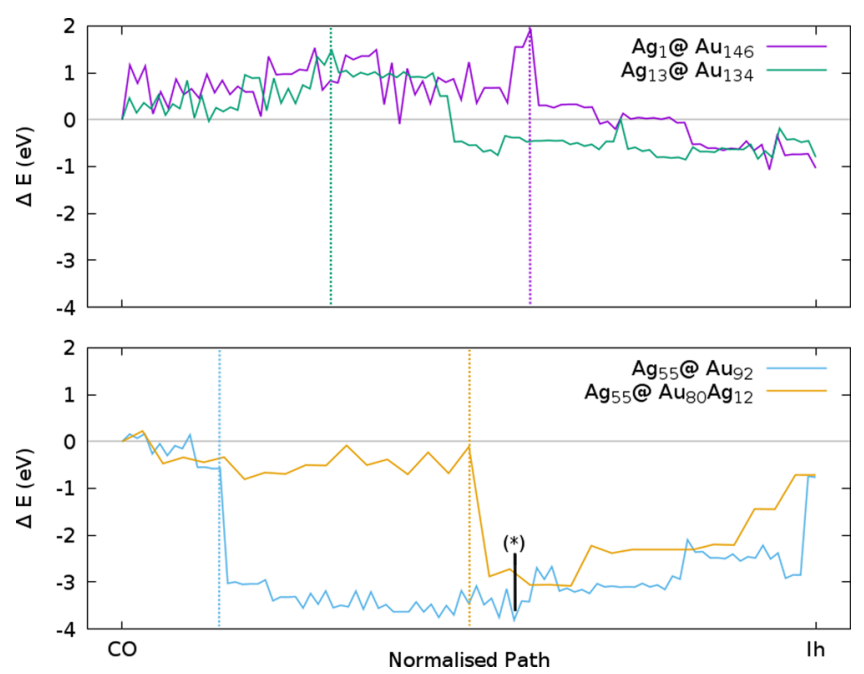

Figure 1. Transition pathways for $\mathrm{CO} \leftrightarrow \mathrm{Ih}$. Top: $\mathrm{Ag}_{1} @ \mathrm{Au}_{146}$ and $\mathrm{Ag}_{13} @ \mathrm{Au}_{134}$; Bottom: $\mathrm{Ag}_{55} @ \mathrm{Au}_{92}$ and $\mathrm{Ag}_{55} @ \mathrm{Au}_{80} \mathrm{Ag}_{12}$. A key is provided in each image, with energies $(\Delta E)$ given relative to that of the $\mathrm{CO}$ motif, that is, akin to $\Delta E(\mathrm{CO} \rightarrow \mathrm{Ih})$. A vertical dashed line in each plot indicates where the transition from CO-like to Ih-like motifs occurs, and $(*)$ indicates the lowest energy rosette-Ih minima encountered for $\mathrm{Ag}_{55} @ \mathrm{Au}_{92}$ and is illustrated in detail in Figure 3.

TO $\rightarrow \mathrm{r}$-Ih $\rightarrow$ Ih transitions. Through the transformation, the $\mathrm{Ag}$ atom is maintained in the central position of the NP, and a ring of $\mathrm{Au}$ atoms forms at the surface to expose a subsurface atom; the lowest energy structure encountered has just one of these rosette indentations. Calculations of the SD and DS pathways for $\mathrm{Ag}_{1} @ \mathrm{Au}_{146}$ give $\Delta E(\mathrm{CO} \rightarrow \mathrm{Ih})$ and $\Delta E(\mathrm{Ih} \rightarrow$ $\mathrm{CO})$ as 1.19 and $2.22 \mathrm{eV}$, which are lower than the $\mathrm{r}$-Ih route and similar to the other values for Ag-doped Au NPs. Analysis of the $r$-Ih transition pathway shows that the initial energy barriers are marginally lower than for the SD mechanism (Figure 2, top) and that the Ih and r-Ih motifs are close in energy.

We expanded our calculations to include larger cores of two and three concentric shells of atoms, corresponding to the central 13 and 55 atoms, respectively. DNEB calculations for $\mathrm{Au}_{13} @ \mathrm{Ag}_{134}$ and $\mathrm{Au}_{55} @ \mathrm{Ag}_{92}$ give $\Delta E(\mathrm{CO} \rightarrow \mathrm{Ih})$ as 0.40 and $0.55 \mathrm{eV}$, with all transformations via the SD mechanism. The reduction of $\Delta E$ for $A_{13} @ A_{134}$ is matched for $\Delta E(\mathrm{Ih} \rightarrow \mathrm{CO})$, where the same compositions have thermodynamic barriers of 3.25 and $2.78 \mathrm{eV}$, respectively, implying that the transition state is reduced in energy.

In contrast, the DNEB pathways are more complicated for $\mathrm{Ag}_{13} @ \mathrm{Au}_{134}$ and $\mathrm{Ag}_{55} @ \mathrm{Au}_{92}$, proceeding via the $\mathrm{r}-\mathrm{Ih}$ intermediates already observed for $\mathrm{Ag}_{1} @ \mathrm{Au}_{146} ; \Delta E(\mathrm{CO} \rightarrow$ Ih) is 1.52 and $3.07 \mathrm{eV}$ for these compositions, respectively, while $\Delta E(\mathrm{Ih} \rightarrow \mathrm{CO})$ is 2.34 and $3.98 \mathrm{eV}$. It is noted that the rate of transformation is determined by the size of the $\mathrm{Ag}$ core: at low $\mathrm{Ag}$ concentration, deformation of the $\mathrm{CO}$ is the timeconsuming process, with the crossover point between $\mathrm{CO}$ - and Ih-like structures higher in energy than for the starting structures (Figure 1, top), whereas at higher Ag concentration, the transition between CO- and Ih-like motifs is via motifs that are lower in energy than the starting structures, and thus $\mathrm{r}$-Ih deformation becomes rate-limiting (Figure 1, bottom). A detailed structural analysis is presented in the Supporting Information, with a distorted TO formed in each case (Figure $3 a)$ before transforming to an $\mathrm{r}$-Ih (Figure $3 \mathrm{~b}$ ) via rotation and stretching similar to the SD mechanism. Dissection of the 

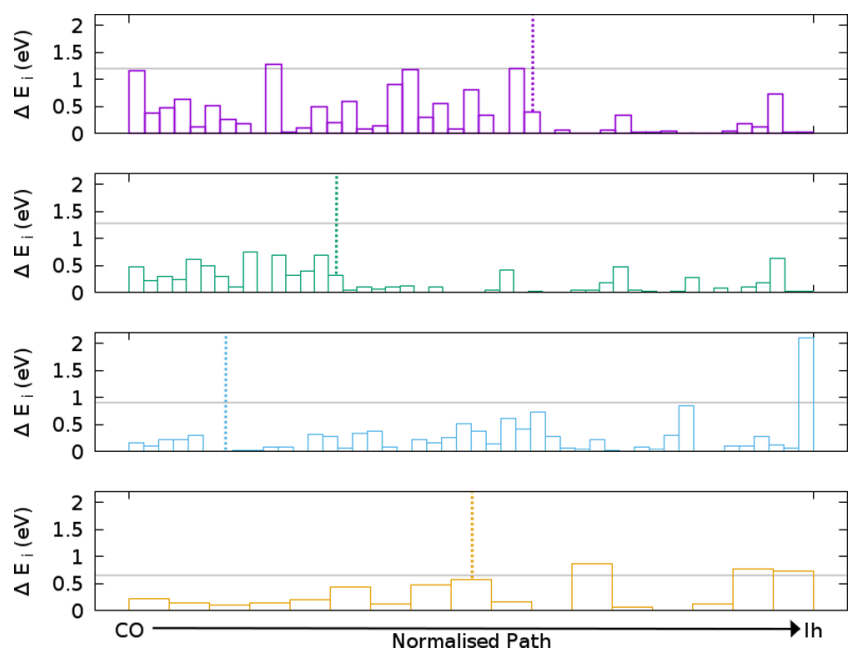

Figure 2. Individual barriers for transition along the $\mathrm{CO} \rightarrow$ Ih pathways in Figure 1. Top: $\mathrm{Ag}_{1} @ \mathrm{Au}_{146}$; Second: $\mathrm{Ag}_{13} @ \mathrm{Au}_{134}$; Third: $\mathrm{Ag}_{55} @ \mathrm{Au}_{92}$; Bottom: $\mathrm{Ag}_{55} @ \mathrm{Au}_{80} \mathrm{Ag}_{12}$. The key is as for Figure 1, with energy barriers $\left(\Delta E_{i}\right)$ given as a function of each individual transition between minima $i$ and $i+1$ in Figure 1. A vertical dashed line in each plot indicates where the transition from CO-like to Ih-like motifs occurs, and the horizontal gray line on each panel represents the barrier height for the more direct SD transition.
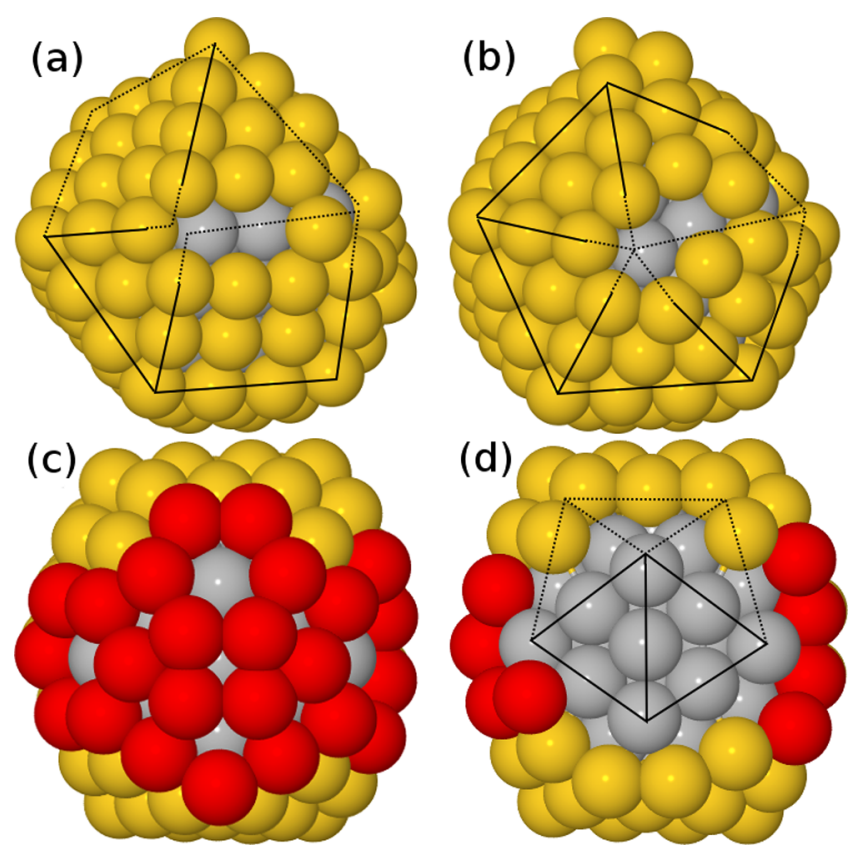

Figure 3. Illustrations of key structures from the transformation of $\mathrm{Ag}_{55} @ \mathrm{Au}_{92}$. Panels a and $\mathrm{b}$ are the CO-like and Ih-like minima either side of the transition marked with vertical dashed blue lines in Figures 1 (bottom) and 2 (second bottom). $\mathrm{Au}$ and $\mathrm{Ag}$ atoms are shown in gold and silver, respectively. Panels $\mathrm{c}$ and $\mathrm{d}$ are the r-Ih local minima identified as the lowest energy arrangement in the transition pathway for $\mathrm{Ag}_{55} @ \mathrm{Au}_{92}$, as marked on Figure 1. Au atoms that form parts of the five-, six- and seven-member rosette rings highlighted in red. In panel c all atoms are included, whereas in panel $\mathrm{d} 16 \mathrm{Au}$ atoms have been removed from the front of the NP to show the underlying Ih symmetry of the Ag core.

lowest energy NP reveals that the Ag core is an Ih motif with five-, six-, and seven-membered rosette rings formed on the NP surface (Figure 3c,d). For $\mathrm{Ag}_{13} @ \mathrm{Au}_{134}$ the low-symmetry r-Ih and high-symmetry Ih are competitive in energy, but for $\mathrm{Ag}_{55} @$ $\mathrm{Au}_{92}$ the $\mathrm{r}$-Ih is substantially lower in energy (Figure 1, bottom).

Interestingly, calculations of the $\mathrm{SD}$ and $\mathrm{DS}$ pathway for $\mathrm{Ag}_{13} @ \mathrm{Au}_{134}$ and $\mathrm{Ag}_{55} @ \mathrm{Au}_{92}$ give $\Delta E(\mathrm{CO} \rightarrow \mathrm{Ih})$ as 1.28 and $0.90 \mathrm{eV}$ and $\Delta E(\mathrm{Ih} \rightarrow \mathrm{CO})$ as 2.08 and $1.66 \mathrm{eV}$, respectively. These direct barriers are lower than via the r-Ih motifs, but the lower initial barriers to form the low-symmetry r-Ih motifs, especially in the case of the $\mathrm{Ag}_{13}$ core, mean that the transformation is taken along (and trapped in) the r-Ih pathway (Figure 2).

Next, we studied the effect of decorating the NP vertices with a secondary species, further increasing the mixing of the Au and $\mathrm{Ag}$ atoms. Such controlled decoration has been achieved for AuPd nanoparticles ${ }^{50}$ and is a logical progression when trying to design chemically bimetallic NPs. Initially, the 12 vertices of pure $\mathrm{Ag}_{147}$ were decorated to create $\mathrm{Ag}_{135} \mathrm{Au}_{12}$, with $\Delta E(\mathrm{CO} \rightarrow$ Ih) and $\Delta E(\mathrm{Ih} \rightarrow \mathrm{CO})$ calculated as 0.35 and $3.50 \mathrm{eV}$, thus showing a decrease and an increase, respectively, compared with $\mathrm{Ag}_{147}$. When the vertices of $\mathrm{Au}_{1} @ \mathrm{Ag}_{146}, \mathrm{Au}_{13} @ \mathrm{Ag}_{134}$, and $\mathrm{Au}_{55} @ \mathrm{Ag}_{92}$ were decorated to give $\mathrm{Au}_{1} @ \mathrm{Ag}_{134} \mathrm{Au}_{12}, \mathrm{Au}_{13} @$ $\mathrm{Ag}_{122} \mathrm{Au}_{12}$, and $\mathrm{Au}_{55} @ \mathrm{Ag}_{80} \mathrm{Au}_{12}$, similar trends were observed: $\Delta E(\mathrm{CO} \rightarrow \mathrm{Ih})$ universally decreases by $\sim 0.1 \mathrm{eV}$ to give 0.38 , 0.27 , and $0.38 \mathrm{eV}$, respectively; for $\Delta E(\mathrm{Ih} \rightarrow \mathrm{CO})$, the transition barriers are $3.38,3.40$, and $2.80 \mathrm{eV}$, which is a slight increase compared with $\mathrm{Ag}_{147}$.

When calculations were repeated for Ag-decorated vertices on Au-rich NPs, rather different behavior was observed: $\mathrm{Au}_{135} \mathrm{Ag}_{12}$ has forward (SD) and backward (DS) barriers of 1.27 and $2.45 \mathrm{eV}$, that is, an increase in 0.13 and $0.12 \mathrm{eV}$, respectively, compared with $\mathrm{Au}_{147}$. For the core@shell arrangements, $\mathrm{Ag}_{1} @ \mathrm{Au}_{134} \mathrm{Ag}_{12}, \mathrm{Ag}_{13} @ \mathrm{Au}_{122} \mathrm{Ag}_{12}$, and $\mathrm{Ag}_{55} @$ $\mathrm{Au}_{80} \mathrm{Ag}_{12}$, only the latter now transforms via the r-Ih pathway. For $A_{1} @ A_{134} A_{12}$ and $A_{13} @ A_{122} A_{12}, \Delta E(C O \rightarrow I h)$ is via the SD pathway and equal to 1.17 and $1.54 \mathrm{eV}$, respectively, but for $\mathrm{Ag}_{55} @ \mathrm{Au}_{80} \mathrm{Ag}_{12}, \Delta E(\mathrm{CO} \rightarrow \mathrm{Ih})$ is $2.37 \mathrm{eV}$, which is a large decrease of $0.70 \mathrm{eV}$ compared with the initial core@shell arrangements (Figure 1, bottom). We note that the transition pathway has significantly fewer steps and that the $\mathrm{TO} \rightarrow \mathrm{r}$-Ih transition is in the center of the pathway, as the complex rotation of $\mathrm{Au}$ atoms on the $\mathrm{r}$-Ih surface is prevented.

A calculation for $\mathrm{Ag}_{55} @ \mathrm{Au}_{80} \mathrm{Ag}_{12}$ transforming via the SD pathway gives a barrier of only $0.64 \mathrm{eV}$ for $\Delta E(\mathrm{CO} \rightarrow \mathrm{Ih})$, and so again we conclude that the $r$-Ih dominates the DNEB calculations due to its low initial barriers and low-energy intermediate minima (Figure 2, bottom). For the backward transition ( $\mathrm{Ih} \rightarrow \mathrm{CO}$ ), $\Delta E$ is 2.20, 2.30, and $3.31 \mathrm{eV}$ for $\mathrm{Ag}_{1} @$ $\mathrm{Au}_{134} \mathrm{Ag}_{12}, \mathrm{Ag}_{13} @ \mathrm{Au}_{122} \mathrm{Ag}_{12}$, and $\mathrm{Ag}_{55} @ \mathrm{Au}_{80} \mathrm{Ag}_{12}$, respectively.

To put our results in the context of experimental investigations, we proceed to free-energy calculations, which we have achieved via both DPS and metadynamics methodologies for pure and core@shell motifs. Calculations using DPS for temperatures $(T)$ of 100 to $500 \mathrm{~K}$ show an almost universal decrease in the free energy of activation for the forward transition, $\Delta F(\mathrm{CO} \rightarrow \mathrm{Ih}):$ a drop of 0.03 to $0.04 \mathrm{eV}$ occurs when going from 0 to $100 \mathrm{~K}$, whereafter the rate of decrease is reduced to $\sim 0.01 \mathrm{eV}$ per $100 \mathrm{~K}$. The only exception is the complicated case of $\mathrm{Ag}_{55} @ \mathrm{Au}_{92}$, where $\Delta \mathrm{F}(\mathrm{CO} \rightarrow \mathrm{Ih})$ increases by $0.04 \mathrm{eV}$ per $100 \mathrm{~K}$ due to the transition states increasing in energy; it is also noted that the r-Ih has high entropy with respect to the $\mathrm{CO}$ and $\mathrm{Ih}$, making it a more favorable minima with increasing $T .^{51}$ The open-ended metadynamics simulations identify various sets of Ih- and CO-like geometries, 

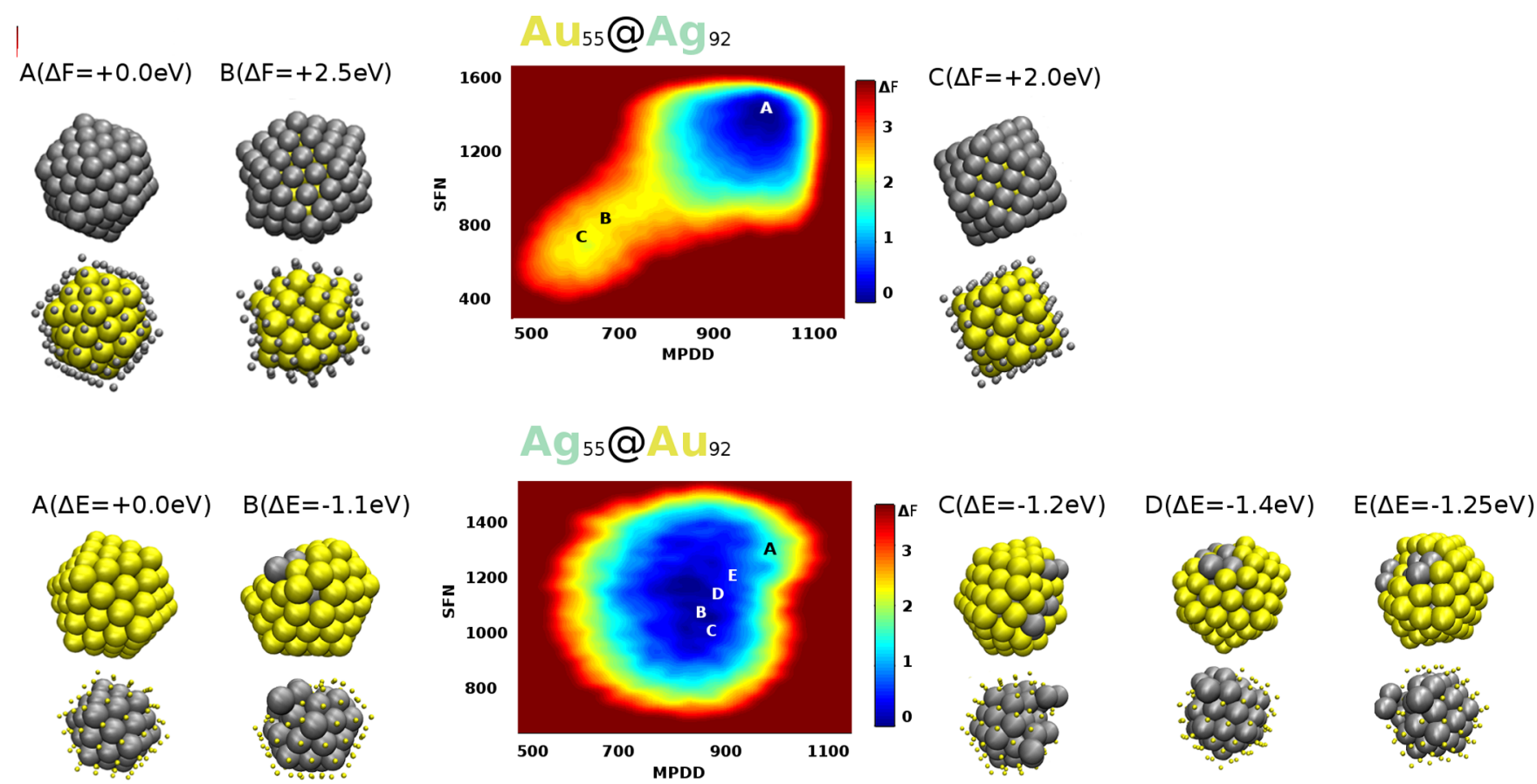

Figure 4. Metadynamics landscape reconstruction in the stacking fault number (SFN) and maximum pair distribution distance (MPDD) collective variable space ${ }^{30}$ for simulations starting in the Ih basin, with free energy $(\Delta F)$ relative to the Ih motif reported at $100 \mathrm{~K}$. Top: $\mathrm{Au}_{55} @ \mathrm{Ag}_{92}$ free-energy landscape connecting Ih (A) and CO (B) basins via a DSD mechanism, as shown by the putative saddle point (C). Bottom: Rosette formation hinders the appearance of the $\mathrm{CO}$ basin in the chosen collective variable space for $\mathrm{Ag}_{55} @ \mathrm{Au}_{92}$. Potential energy $(\Delta E)$ of the most relevant minima is reported to show their relative stability against the Ih motif. Morphologies are displayed using atomic models with gray and yellow spheres representing silver and gold atoms, respectively.

similar to those observed in the DNEB calculations (Figure 4, bottom). In general, the metadynamics simulations give more rapidly decreasing barriers, with $\Delta F(\mathrm{CO} \rightarrow \mathrm{Ih})$ reduced from $0.4 \mathrm{eV}$ at $50 \mathrm{~K}$ to $0.1 \mathrm{eV}$ at $\mathrm{T}=150 \mathrm{~K}$ for all Ag-rich systems, including those with Au-decorated vertices. More detailed calculations were also pursued for single $\mathrm{Au}$ dopants in $\mathrm{Ag}_{147}$, that is, $\mathrm{Au}_{1} \mathrm{Ag}_{146}$, with the position of the $\mathrm{Au}$ atoms altering the barriers by at most $0.1 \mathrm{eV}$, and all barriers inversely correlated with $T$.

For the backward transition, DPS shows that $\Delta F(\mathrm{Ih} \rightarrow \mathrm{CO})$ decreases at varying rates depending on the composition: For $\mathrm{Ag}_{147}, \Delta F(\mathrm{Ih} \rightarrow \mathrm{CO})$ decreases by $0.02 \mathrm{eV}$ per $100 \mathrm{~K}$, but for $\mathrm{Au}_{147}$ the rate is doubled to $0.04 \mathrm{eV}$ per $100 \mathrm{~K}$. For Ag-rich core@shell motifs, that is, with an Ag shell, the rate of decrease remains at $0.02 \mathrm{eV}$ per $100 \mathrm{~K}$, while for Au-rich motifs the clear anomaly is $\mathrm{Ag}_{55} @ \mathrm{Au}_{92}$, for which $\Delta \mathrm{F}(\mathrm{Ih} \rightarrow \mathrm{CO})$ initially decreases from $3.98(0 \mathrm{~K})$ to $3.94 \mathrm{eV}(300 \mathrm{~K})$ and then increases back up to $3.98 \mathrm{eV}(500 \mathrm{~K})$ due to the energetic variation in TO structures close to the $\mathrm{CO}$ motif. Again, analysis with metadynamics gives slightly lower barriers than DPS, with Ag-rich systems displaying $\Delta F(\mathrm{Ih} \rightarrow \mathrm{CO})=2.85 \pm$ $0.15 \mathrm{eV}$ at $50 \mathrm{~K}$, and these barrier heights are maintained at 100 and $150 \mathrm{~K}$ (Figure 4, bottom). For single atom dopants in $\mathrm{Au}_{1} \mathrm{Ag}_{146}$, the barriers are calculated to be 2.8 to $3.0 \mathrm{eV}$ at $50 \mathrm{~K}$ and steady in this energy range up to $150 \mathrm{~K}$.

The overall differences in barriers calculated using DPS and metadynamics are deemed insignificant with the small underestimate from metadynamics probably due to the effect of reduced dimensionality. The identical pathways and similar barrier heights identified by both methods, which include the rIh-assisted transformations for $\mathrm{Ag} @ \mathrm{Au}$, show that anharmonic effects do not play a significant role in the SD path. In addition, the anharmonic contributions can lower energetic barriers, which explains part of the difference between our DPS and metadynamics results. Importantly, an increase in $T$ does not change the mechanism of structural transition for either calculation method, with chemical composition and ordering having a much stronger influence on barriers.

In conclusion, we have shown that the transition barrier between high-symmetry geometric motifs for bimetallic NPs is not merely a linear interpolation between systems but instead is strongly dependent on chemical arrangements. In particular, we have highlighted the complexity of the potential and freeenergy landscapes for systems where there are low-symmetry geometric motifs energetically competitive with the "highsymmetry" arrangements often pursued by theoretical and experimental scientists alike. In addition, we have also shown that the transition pathway can be controlled via careful construction of the NP with respect to stoichiometry and chemical arrangements, specifically by the use of vertex-doping to restrict surface-based structural transformations. Our results offer potential for future work in bespoke nanocatalyst and nanoplasmonic design, where structural stability due to specific geometric features, such as surface facets and vertex decoration, could be facilitated by careful consideration of the composition of the NPs in question.

\section{ASSOCIATED CONTENT}

\section{S Supporting Information}

The Supporting Information is available free of charge on the ACS Publications website at DOI: 10.1021/acs.jpclett.6b02181.

Detailed structural analysis for the non-DSD transitions between $\mathrm{CO}$ and $\mathrm{Ih}$ of $\mathrm{Ag}_{1} @ \mathrm{Au}_{146}, \mathrm{Ag}_{13} @ \mathrm{Au}_{134}, \mathrm{Ag}_{55} @$ $\mathrm{Au}_{92}$, and $\mathrm{Ag}_{55} @ \mathrm{Au}_{80} \mathrm{Ag}_{12}$. (PDF) 


\section{AUTHOR INFORMATION}

\section{Corresponding Authors}

*F.B.: E-mail: francesca.baletto@kcl.ac.uk.

*A.J.L.: E-mail: a.logsdail@ucl.ac.uk.

Notes

The authors declare no competing financial interest.

\section{ACKNOWLEDGMENTS}

A.J.L. acknowledges the Ramsay Memorial Trust and University College London for the provision of a Ramsay Fellowship. A.J.L. and C.R.A.C. acknowledge financial support from the EPSRC, U.K. (Grant Reference: EP/IO30662/1), as does K.R. (Grant Reference ER/M506357/1) and F.B. (Grant References: EP/J010812/1 and EP/G003146/1), the latter of which is through Critical Mass Grant No. 408. K.R. and F.B. also acknowledge financial support from the Royal Society (RG 120207). We acknowledge D. Wales for his support using the OPTIM software package, N. Dimitratos and A. A. Sokol for several stimulating and useful conversations, and Jörg Sassmanshausen for continued IT support. A.G., C.R.A.C., and A.J.L. acknowledge the use of the UCL and ARCHER high-performance computing facilities, and associated support services, with the latter supported via their membership of the UK HPC Materials Chemistry Consortium (EP/L000202).

\section{REFERENCES}

(1) Johnston, R. L. Atomic and Molecular Clusters; Taylor and Francis: London, 2002.

(2) Heiz, U.; Landman, U. Nanocatalysis; Springer-Verlag: Berlin, 2007.

(3) Ray, P. C. Size and Shape Dependent Second Order Nonlinear Optical Properties of Nanomaterials and Their Application in Biological and Chemical Sensing. Chem. Rev. 2010, 110, 5332-5365.

(4) Liu, J. P.; Fullerton, E.; Gutfleisch, O.; Sellmyer, D. J. Nanoscale Magnetic Materials and Applications; Springer: New York, 2009.

(5) Corain, B.; Shemid, G.; Toshima, N. Metal Nanoclusters in Catalysis and Materials Science: The Issue of Size Control; Elsevier: Amsterdam, The Netherlands, 2008.

(6) Asara, G. G.; Paz-Borbón, L. O.; Baletto, F. "Get in Touch and Keep in Contact": Interface Effect on the Oxygen Reduction Reaction (ORR) Activity for Supported PtNi Nanoparticles. ACS Catal. 2016, 6, 4388-4393.

(7) Baletto, F.; Ferrando, R. Doped golden fullerene cages. Phys. Chem. Chem. Phys. 2015, 17, 28256-28261.

(8) Ferrando, R.; Jellinek, J.; Johnston, R. L. Nanoalloys: From Theory to Applications of Alloy Clusters and Nanoparticles. Chem. Rev. 2008, 108, 845-910.

(9) Li, Z. Y.; Young, N. P.; Di Vece, M. D.; Palomba, S.; Palmer, R. E.; Bleloch, A. L.; Curley, B. C.; Johnston, R. L.; Jiang, J.; Yuan, J. Three-dimensional Atomic-scale Structure of Size-selected Gold Nanoclusters. Nature 2008, 451, 46-48.

(10) José-Yacamán, M.; Ascencio, J. A.; Liu, H. B.; Gardea-Torresdey, J. Structure shape and stability of nanometric sized particles. J. Vac. Sci. Technol., B: Microelectron. Process. Phenom. 2001, 19, 1091-1103.

(11) Palmer, R. E.; Pratontep, S.; Boyen, H. G. Nanostructured Surfaces from Size-selected Clusters. Nat. Mater. 2003, 2, 443-448.

(12) Yin, Z.; Zhang, Y.; Chen, K.; Li, J.; Li, W.; Tang, P.; Zhao, H.; Zhu, Q.; Bao, X.; Ma, D. Monodispersed bimetallic PdAg nanoparticles with twinned structures: Formation and enhancement for the methanol oxidation. Sci. Rep. 2014, 4, 4288.

(13) Lu, J.; Low, K.-B.; Lei, Y.; Libera, J. A.; Nicholls, A.; Stair, P. C.; Elam, J. W. Toward atomically-precise synthesis of supported bimetallic nanoparticles using atomic layer deposition. Nat. Commun. 2014, 5, 3264.

(14) Strasser, P.; Koh, S.; Anniyev, T.; Greeley, J.; More, K.; Yu, C.; Liu, Z.; Kaya, S.; Nordlund, D.; Ogasawara, H.; et al. Lattice-strain control of the activity in dealloyed core-shell fuel cell catalysts. Nat. Chem. 2010, 2, 454-460.

(15) Wells, D. M.; Rossi, G.; Ferrando, R.; Palmer, R. E. Metastability of the atomic structures of size-selected gold nanoparticles. Nanoscale 2015, 7, 6498-6503.

(16) Baletto, F.; Ferrando, R. Structural properties of nanoclusters: Energetic, thermodynamic, and kinetic effects. Rev. Mod. Phys. 2005, 77, 371-423.

(17) Haruta, M.; Tsubota, S.; Kobayashi, T.; Kageyama, H.; Genet, M. J.; Delmon, B. Low-Temperature Oxidation of $\mathrm{CO}$ over Gold Supported on $\mathrm{TiO}_{2}, \alpha-\mathrm{Fe}_{2} \mathrm{O}_{3}$, and $\mathrm{Co}_{3} \mathrm{O}_{4}$. J. Catal. 1993, 144, 175192.

(18) Calle-Vallejo, F.; Loffreda, D.; Koper, M. T.; Sautet, P. Introducing structural sensitivity into adsorption-energy scaling relations by means of coordination numbers. Nat. Chem. 2015, 7, 403-410.

(19) Calle-Vallejo, F.; Tymoczko, J.; Colic, V.; Vu, Q. H.; Pohl, M. D.; Morgenstern, K.; Loffreda, D.; Sautet, P.; Schuhmann, W.; Bandarenka, A. S. Finding optimal surface sites on heterogeneous catalysts by counting nearest neighbors. Science 2015, 350, 185-189.

(20) Viñes, F.; Gomes, J. R.; Illas, F. Understanding the reactivity of metallic nanoparticles: beyond the extended surface model for catalysis. Chem. Soc. Rev. 2014, 43, 4922-4939.

(21) Rogers, S. M.; Catlow, C. R. A.; Chan-Thaw, C. E.; Gianolio, D.; Gibson, E. K.; Gould, A. L.; Jian, N.; Logsdail, A. J.; Palmer, R. E.; Prati, L.; et al. Tailoring Gold Nanoparticle Characteristics and the Impact on Aqueous-Phase Oxidation of Glycerol. ACS Catal. 2015, 5, 4377-4384.

(22) Dessens-Félix, M.; Pacheco-Contreras, R.; Barcaro, G.; Sementa, L.; Fortunelli, A.; Posada-Amarillas, A. Structural Motifs of Bimetallic Pt101-x Au x Nanoclusters. J. Phys. Chem. C 2013, 117, 2096720974.

(23) Bochicchio, D.; Negro, F.; Ferrando, R. Competition between structural motifs in gold-platinum nanoalloys. Comput. Theor. Chem. 2013, 1021, 177-182.

(24) Wang, Z. W.; Palmer, R. E. Direct Atomic Imaging and Dynamical Fluctuations of the Tetrahedral Au20 Cluster. Nanoscale 2012, 4, 4947-4949.

(25) Wang, Z. W.; Palmer, R. E. Determination of the ground-state atomic structures of size-selected Au nanoclusters by electron-beaminduced transformation. Phys. Rev. Lett. 2012, 108, 245502.

(26) Lipscomb, W. N. Framework Rearrangement in Boranes and Carboranes. Science 1966, 153, 373-378.

(27) Baletto, F.; Mottet, C.; Ferrando, R. Reentrant morphology transition in the growth of free silver nanoclusters. Phys. Rev. Lett. 2000, 84, 5544.

(28) Baletto, F.; Mottet, C.; Ferrando, R. Microscopic mechanisms of the growth of metastable silver icosahedra. Phys. Rev. B: Condens. Matter Mater. Phys. 2001, 63, 155408.

(29) Aprà, E.; Baletto, F.; Ferrando, R.; Fortunelli, A. Amorphization Mechanism of Icosahedral Metal Nanoclusters. Phys. Rev. Lett. 2004, 93, 065502.

(30) Pavan, L.; Rossi, K.; Baletto, F. Metallic nanoparticles meet metadynamics. J. Chem. Phys. 2015, 143, 184304.

(31) Mottet, C.; Rossi, G.; Baletto, F.; Ferrando, R. Single impurity effect on the melting of nanoclusters. Phys. Rev. Lett. 2005, 95, 035501.

(32) Gould, A. L.; Logsdail, A. J.; Catlow, C. R. A. Influence of Composition and Chemical Arrangement on the Kinetic Stability of 147-Atom Au-Ag Bimetallic Nanoclusters. J. Phys. Chem. C 2015, 119, 23685-23697.

(33) Chen, F.; Johnston, R. L. Martensitic transformations in Ag-Au bimetallic core-shell nanoalloys. Appl. Phys. Lett. 2008, 92, 023112.

(34) Chen, F.; Curley, B. C.; Rossi, G.; Johnston, R. L. Structure, melting, and thermal stability of 55 atom Ag-Au nanoalloys. J. Phys. Chem. C 2007, 111, 9157-9165.

(35) Mackay, A. L. A dense non-crystallographic packing of equal spheres. Acta Crystallogr. 1962, 15, 916-918. 
(36) Chen, F.; Li, Z. Y.; Johnston, R. L. Surface reconstruction precursor to melting in $\mathrm{Au}[\mathrm{sub}$ 309] clusters. AIP Adv. 2011, 1, 032105.

(37) Baletto, F.; Ferrando, R.; Fortunelli, A.; Montalenti, F.; Mottet, C. Crossover among structural motifs in transition and noble-metal clusters. J. Chem. Phys. 2002, 116, 3856-3863.

(38) Logsdail, A. J.; Johnston, R. L. Interdependence of Structure and Chemical Order in High Symmetry (PdAu) ${ }_{N}$ Nanoclusters. RSC Adv. 2012, 2, 5863-5869.

(39) Cleri, F.; Rosato, V. Tight-binding potentials for transition metals and alloys. Phys. Rev. B: Condens. Matter Mater. Phys. 1993, 48, $22-33$.

(40) Gould, A.; Heard, C.; Logsdail, A.; Catlow, C. Segregation effects on the properties of (AuAg) 147. Phys. Chem. Chem. Phys. 2014, 16, 21049-21061.

(41) Trygubenko, S. A.; Wales, D. J. A doubly nudged elastic band method for finding transition states. J. Chem. Phys. 2004, 120, 20822094.

(42) Wales, D. Energy Landscapes: Applications to Clusters, Biomolecules and Glasses; Cambridge University Press, 2003.

(43) Wales, D. J. Energy landscapes: calculating pathways and rates. Int. Rev. Phys. Chem. 2006, 25, 237-282.

(44) Wales, D. J. Discrete path sampling. Mol. Phys. 2002, 100, 3285-3305.

(45) Wales, D. J. Some further applications of discrete path sampling to cluster isomerization. Mol. Phys. 2004, 102, 891-908.

(46) Trygubenko, S. A.; Wales, D. J. Kinetic analysis of discrete path sampling stationary point databases. Mol. Phys. 2006, 104, 1497-1507.

(47) Laio, A.; Parrinello, M. Escaping free-energy minima. Proc. Natl. Acad. Sci. U. S. A. 2002, 99, 12562-12566.

(48) Laio, A.; Gervasio, F. L. Metadynamics: a method to simulate rare events and reconstruct the free energy in biophysics, chemistry and material science. Rep. Prog. Phys. 2008, 71, 126601.

(49) Gale, J. D.; Rohl, A. L. The General Utility Lattice Program (GULP). Mol. Simul. 2003, 29, 291-341.

(50) Zhang, H.; Watanabe, T.; Okumura, M.; Haruta, M.; Toshima, N. Catalytically Highly Active Top Gold Atom on Palladium Nanocluster. Nat. Mater. 2011, 11, 49-52.

(51) Doye, J. P. K.; Calvo, F. Entropic Effects on the Size Dependence of Cluster Structure. Phys. Rev. Lett. 2001, 86, 35703573. 